\title{
TWO NEW PROOFS OF LERCH'S FUNCTIONAL EQUATION
}

\author{
BRUCE C. BERNDT
}

ABSTRACT.

One bright Sunday morning I went to church,

And there I met a man named Lerch.

We both did sing in jubilation,

For he did show me a new equation.

Two simple derivations of the functional equation of

$$
\sum_{n=0}^{\infty} \exp [2 \pi i n x](n+a)^{-s}
$$

are given. The original proof is due to Lerch.

If $x$ is real and $0<a \leqq 1$, define

$$
\varphi(x, a, s)=\sum_{n=0}^{\infty} \exp [2 \pi i n x](n+a)^{-s},
$$

where $\sigma=\operatorname{Re} s>1$ if $x$ is an integer, and $\sigma>0$ otherwise. Note that $\varphi(0, a, s)=\zeta(s, a)$, the Hurwitz zeta-function. Furthermore, if $a=1$, $\varphi(0,1, s)=\zeta(s)$, the Riemann zeta-function.

In 1887, Lerch [1] derived the following functional equation for $\varphi(x, a, s)$.

THEOREM. Let $0<x<1$. Then $\varphi(x, a, s)$ has an analytic continuation to the entire complex plane and is an entire function of s. Furthermore, for all s,

$$
\begin{aligned}
& \varphi(x, a, 1-s)=(2 \pi)^{-s} \Gamma(s) \\
& \cdot\left\{\exp \left[\frac{1}{2} \pi i s-2 \pi i a x\right] \varphi(-a, x, s)\right. \\
&\left.\quad+\exp \left[-\frac{1}{2} \pi i s+2 \pi i a(1-x)\right] \varphi(a, 1-x, s)\right\} .
\end{aligned}
$$

Lerch's proof [1] of (1) depends upon the evaluation of a certain loop integral. Our objective here is to give two simple, new proofs of (1). The first proof uses contour integration; the second employs the EulerMaclaurin summation formula. By slight variations of our methods, one can derive the corresponding result, namely Hurwitz's formula, for $\varphi(0, a, s)=\zeta(s, a)$.

Received by the editors March 3, 1971 and, in revised form, June 18, 1971.

AMS 1969 subject classifications. Primary 1041; Secondary 1040.

Key words and phrases. Lerch's zeta-function, Hurwitz zeta-function, Riemann zetafunction, functional equation, Euler-Maclaurin summation formula.

(c) American Mathematical Society 1972 
First Proof. Assume that $s>1$ is real. With the aid of Euler's integral representation for $\Gamma(s)$, it is easy to show that $[1, \mathrm{pp} .19-20]$

$$
\Gamma(s) \varphi(x, a, s)=\int_{0}^{\infty} \frac{\exp [(1-a) u-2 \pi i x]}{\exp [u-2 \pi i x]-1} u^{s-1} d u .
$$

If we put $x=b+\frac{1}{2}$, then $|b|<\frac{1}{2}$. Define

$$
F(z)=\frac{\pi \exp [2 \pi i b z]}{(z+a)^{s} \sin (\pi z)},
$$

where the principal branch of $(z+a)^{s}$ is chosen. Choose $c$ so that $-a<c<0$. If $m$ is a positive integer, let $C_{m}$ denote the positively oriented contour consisting of the right half of the circle with center $(c, 0)$ and radius $m+\frac{1}{2}-c$ together with the vertical diameter through $(c, 0)$. By the residue theorem,

$$
\frac{1}{2 \pi i} \int_{C_{m}} F(z) d z=\sum_{n=0}^{m} \exp [2 \pi i n x](n+a)^{-s} .
$$

Let $\Gamma_{m}$ denote the circular part of $C_{m}$. Since $|b|<\frac{1}{2}$, there is a constant $M$, independent of $m$, such that for $z$ on $\Gamma_{m}, m \geqq 1$,

Hence,

$$
\left|\frac{\exp [2 \pi i b z]}{\sin (\pi z)}\right| \leqq M
$$

$$
\left|\int_{\Gamma_{m}} F(z) d z\right| \leqq \frac{\pi^{2}\left(m+\frac{3}{2}\right) M}{\left(m+\frac{1}{2}\right)^{s}}
$$

which tends to 0 as $m$ tends to $\infty$ since $s>1$. Thus, upon letting $m$ tend to $\infty$ in (3), we find that

$$
\begin{aligned}
\varphi(x, a, s)= & -\frac{1}{2 \pi i} \int_{c-i \infty}^{c+i \infty} F(z) d z \\
= & \int_{c}^{c+i \infty} \frac{\exp [2 \pi i b z-\pi i z]}{(z+a)^{s}(\exp [-2 \pi i z]-1)} d z \\
& +\int_{c}^{c-i \infty} \frac{\exp [2 \pi i b z+\pi i z]}{(z+a)^{s}(\exp [2 \pi i z]-1)} d z .
\end{aligned}
$$

We observe that the integrals in (4) converge uniformly in any compact set of the $s$-plane since $|b|<\frac{1}{2}$. Hence, (4) shows that $\varphi(x, a, s)$ can be analytically continued to an entire function of $s$.

Now suppose that $-1<s<0$. We wish to let $c$ approach $-a$ in (4). In a neighborhood of $z=-a$, we have

$$
\left|\frac{\exp [2 \pi i b z \pm \pi i z]}{(z+a)^{s}(\exp [ \pm 2 \pi i z]-1)}\right| \leqq A|z+a|^{-s-1} \leqq A|y|^{-s-1}
$$


where $A$ is some positive number. Since $-1<s<0$,

$$
\int_{0}^{\mp 1}|y|^{-s-1} d y<\infty
$$

Hence, the two integrals on the right side of (4) converge uniformly on $-a \leqq c \leqq-a+\varepsilon$, for any number $\varepsilon>0$. We may then let $c$ tend to $-a$ in (4) to obtain

$$
\begin{aligned}
\varphi(x, a, s)= & i \exp \left[-\frac{1}{2} \pi i s-2 \pi i a b+\pi i a\right] \\
& \cdot \int_{0}^{\infty} \frac{\exp [-2 \pi b y+\pi y]}{y^{s}(\exp [2 \pi y+2 \pi i a]-1)} d y \\
& -i \exp \left[\frac{1}{2} \pi i s-2 \pi i a b-\pi i a\right] \\
& \cdot \int_{0}^{\infty} \frac{\exp [2 \pi b y+\pi y]}{y^{s}(\exp [2 \pi y-2 \pi i a]-1)} d y .
\end{aligned}
$$

If we make the substitutions $u=2 \pi y$ and $b=x-\frac{i}{2}$ and replace $s$ by $1-s$, the above becomes, for $s>1$,

$$
\begin{aligned}
\varphi(x, a, 1-s)= & \exp \left[\frac{1}{2} \pi i s-2 \pi i a(x-1)\right](2 \pi)^{-s} \\
& \cdot \int_{0}^{\infty} \frac{\exp [-u(x-1)] u^{s-1}}{\exp [u+2 \pi i a]-1} d u \\
& +\exp \left[-\frac{1}{2} \pi i s-2 \pi i a x\right](2 \pi)^{-s} \\
& \cdot \int_{0}^{\infty} \frac{\exp [u x] u^{s-1}}{\exp [u-2 \pi i a]-1} d u
\end{aligned}
$$

If we now use (2), (1) immediately follows for $s>1$. By analytic continuation, (1) is valid for all $s$.

Second Proof. Let $f$ have a continuous first derivative on $[c, m]$, where $m$ is a positive integer. Then we have the Euler-Maclaurin summation formula,

$$
\begin{aligned}
\sum_{c<n \leqq m} f(n)= & \int_{c}^{m} f(u) d u+\frac{1}{2} f(m)+\left(c-[c]-\frac{1}{2}\right) f(c) \\
& +\int_{c}^{m}\left(u-[u]-\frac{1}{2}\right) f^{\prime}(u) d u .
\end{aligned}
$$

Put $c=1-a$ and $f(u)=(u+a)^{-s} \exp (2 \pi i u x)$, where $\sigma>0$. Then, upon letting $m$ tend to $\infty$ in (5), we obtain

$$
\begin{aligned}
\varphi(x, a, s)-a^{-s}= & \int_{1-a}^{\infty}(u+a)^{-s} \exp [2 \pi i u x] d u \\
& +\left(\frac{1}{2}-a\right) \exp [2 \pi i x(1-a)] \\
& -s \int_{1-a}^{\infty} \frac{u-[u]-\frac{1}{2}}{(u+a)^{s+1}} \exp [2 \pi i u x] d u \\
& +2 \pi i x \int_{1-a}^{\infty} \frac{u-[u]-\frac{1}{2}}{(u+a)^{s}} \exp [2 \pi i u x] d u
\end{aligned}
$$


The three integrals on the right side of (6) all converge for $\sigma>0$ by Dirichlet's test.

First, assume that $0<\sigma<1$. Since

$$
[u]-u+\frac{1}{2}=\sum_{n=1}^{\infty} \frac{\sin (2 \pi n u)}{\pi n}
$$

if $u$ is not an integer, we have formally

$$
\begin{aligned}
& \int_{-a}^{\infty} \frac{u-[u]-\frac{1}{2}}{(u+a)^{s}} \exp [2 \pi i u x] d u \\
& \quad=-\frac{1}{\pi} \int_{0}^{\infty} u^{-s} \exp [2 \pi i(u-a) x] \sum_{n=1}^{\infty} \frac{1}{n} \sin (2 \pi n\{u-a\}) d u \\
& \begin{aligned}
(8) \quad=\frac{\exp [-2 \pi i a x]}{2 \pi i} \sum_{n=1}^{\infty} \frac{1}{n}\left\{\exp [2 \pi i n a] \int_{0}^{\infty} u^{-s} \exp [2 \pi i u(x-n)] d u\right. \\
\left.-\exp [-2 \pi i n a] \int_{0}^{\infty} u^{-s} \exp [2 \pi i u(x+n)] d u\right\}
\end{aligned}
\end{aligned}
$$

We must justify the inversion in order of summation and integration. Since the Fourier series in (7) is boundedly convergent, the inversion is justified if we integrate over $(0, b)$, where $b$ is any finite number [2, p. 41]. We need then only show that, for $0<\sigma<1$,

$$
\begin{aligned}
\lim _{b \rightarrow \infty} \sum_{n=1}^{\infty} \frac{1}{n}\left\{\exp [2 \pi i n a] \int_{b}^{\infty} u^{-s} \exp [2 \pi i u(x-n)] d u\right. \\
\left.-\exp [-2 \pi i n a] \int_{b}^{\infty} u^{-s} \exp [2 \pi i u(x+n)] d u\right\}=0 .
\end{aligned}
$$

Upon an integration by parts,

$$
\begin{aligned}
\int_{b}^{\infty} u^{-s} \exp [2 \pi i u(x-n)] d u & =O\left(b^{-\sigma} / n\right) \\
& +\frac{s}{2 \pi i(x-n)} \int_{b}^{\infty} u^{-s-1} \exp [2 \pi i u(x-n)] d u=O\left(b^{-\sigma} / n\right),
\end{aligned}
$$

as $b$ tends to $\infty$. By the same argument we obtain the same $O$-estimate for the integrals involving $\exp \{2 \pi i u(x+n)\}$. Hence, (9) now easily follows.

Now, if $0<\sigma<1$ and $d \neq 0$ is real, we have [2, pp. 107-1c.9]

$$
\int_{0}^{\infty} u^{-s} \exp [i d u] d u=\Gamma(1-s)|d|^{s-1} \exp \left[\frac{1}{2} \pi i(1-s) \operatorname{sgn} d\right]
$$


Thus, (8) becomes

$$
\begin{aligned}
& \int_{-a}^{\infty} \frac{u-[u]-\frac{1}{2}}{(u+a)^{s}} \exp [2 \pi i u x] d u \\
&=-(2 \pi)^{s-2} \Gamma(1-s) \exp \left[\frac{1}{2} \pi i s-2 \pi i a x\right] \\
& \cdot \sum_{n=1}^{\infty}\left\{\frac{\exp [2 \pi i n a]}{n(n-x)^{1-s}}+\frac{\exp [-\pi i s-2 \pi i n a]}{n(n+x)^{1-s}}\right\} .
\end{aligned}
$$

Using (10) again, we have, for $0<\sigma<1$,

$$
\begin{aligned}
\int_{-a}^{\infty}(u+a)^{-s} \exp [2 \pi i u x] & d u \\
= & \exp [-2 \pi i a x] \int_{0}^{\infty} u^{-s} \exp [2 \pi i u x] d u \\
= & \Gamma(1-s)(2 \pi x)^{s-1} \exp \left[\frac{1}{2} \pi i(1-s)-2 \pi i a x\right] .
\end{aligned}
$$

Hence, substituting (11) and (12) into (6), we obtain, for $0<\sigma<1$,

$$
\begin{aligned}
& \varphi(x, a, x)-a^{-s} \\
& =\Gamma(1-s)(2 \pi x)^{s-1} \exp \left[\frac{1}{2} \pi i(1-s)-2 \pi i a x\right] \\
& \quad-\int_{-a}^{1-a}(u+a)^{-s} \exp [2 \pi i u x]\left\{1+2 \pi i x\left(u-[u]-\frac{1}{2}\right)\right\} d u \\
& \quad+\left(\frac{1}{2}-a\right) \exp [2 \pi i x(1-a)]-s \int_{1-a}^{\infty} \frac{u-[u]-\frac{1}{2}}{(u+a)^{s+1}} \exp [2 \pi i u x] d u \\
& \quad+x(2 \pi)^{s-1} \Gamma(1-s) \exp \left[\frac{1}{2} \pi i(s-1)-2 \pi i a x\right] \\
& \quad \cdot \sum_{n=1}^{\infty}\left\{\frac{\exp [2 \pi i n a]}{n(n-x)^{1-s}}+\frac{\exp [-\pi i s-2 \pi i n a]}{n(n+x)^{1-s}}\right\} .
\end{aligned}
$$

We next observe that the infinite series on the right side of (13) converges absolutely and uniformly on any compact subset of the strip $-1<\sigma<1$. By Dirichlet's test, the integrals on the right side of (13) converge uniformly on any compact subset of the strip $-1<\sigma<1$. Hence, by analytic continuation, (13) is valid for $-1<\sigma<1$. Assume now that $-1<\sigma<0$. $\operatorname{Re}$ placing $s$ by $s+1$ in (11), we have

$$
\begin{aligned}
\int_{-a}^{\infty} \frac{u-[u]-\frac{1}{2}}{(u+a)^{s+1}} \exp [2 \pi i u x] d u & \\
= & (2 \pi)^{s-1} \Gamma(-s) \exp \left[\frac{1}{2} \pi i(s-1)-2 \pi i a x\right] \\
& \cdot \sum_{n=1}^{\infty}\left\{\frac{\exp [2 \pi i n a]}{n(n-x)^{-s}}-\frac{\exp [-i \pi s-2 \pi i n a]}{n(n+x)^{-s}}\right\} .
\end{aligned}
$$


Substitute (14) into (13), use the functional equation of $\Gamma(s)$, and observe that $x /\{n(n-x)\}+1 / n=1 /(n-x)$ and $x /\{n(n+x)\}-1 / n=-1 /(n+x)$. For $-1<\sigma<0$ we arrive at

$$
\begin{aligned}
\varphi(x, a, s) & -a^{-s} \\
= & \Gamma(1-s)(2 \pi x)^{s-1} \exp \left[\frac{1}{2} \pi i(1-s)-2 \pi i a x\right] \\
& -\int_{-a}^{1-a}(u+a)^{-s} \exp [2 \pi i u x] \\
& \cdot\left\{1+2 \pi i x\left(u-[u]-\frac{1}{2}\right)-s(u+a)^{-1}\left(u-[u]-\frac{1}{2}\right)\right\} d u \\
+ & \left(\frac{1}{2}-a\right) \exp [2 \pi i x(1-a)] \\
+ & (2 \pi)^{s-1} \Gamma(1-s) \exp \left[\frac{1}{2} \pi i(s-1)-2 \pi i a x\right] \\
& \cdot\left\{\sum_{n=1}^{\infty} \frac{\exp [2 \pi i n a]}{(n-x)^{1-s}}-\sum_{n=1}^{\infty} \frac{\exp [-\pi i s-2 \pi i n a]}{(n+x)^{1-s}}\right\} .
\end{aligned}
$$

Observe that the first expression on the right side of (15) corresponds to the term $n=0$ for the second series on the right side of (15). In the first series replace $n$ by $n+1$. By an elementary calculation the second expression on the right side of (15) is seen to be

$$
-a^{-s}-\left(\frac{1}{2}-a\right) \exp [2 \pi i x(1-a)] .
$$

Upon these simplifications, (15) now becomes, for $-1<\sigma<0$,

$$
\begin{aligned}
\varphi(x, a, x) \\
=(2 \pi)^{s-1} \Gamma(1-s) \exp \left[\frac{1}{2} \pi i(s-1)-2 \pi i a x\right] \\
\quad \cdot\{\exp [2 \pi i a] \varphi(a, 1-x, 1-s)-\exp [-\pi i s] \varphi(-a, x, 1-s)\} .
\end{aligned}
$$

By analytic continuation (16) is valid for all $s$. Now replace $s$ by $1-s$ in (16) to obtain (1).

\section{REFERENCES}

1. M. Lerch, Note sur la fonction $\mathfrak{R}(w, x, s)=\sum_{k=0}^{\infty} e^{2 k \pi i x} /(w+k)^{s}$, Acta Math. 11 (1887), 19-24.

2. E. C. Titchmarsh, The theory of functions, 2nd ed., Oxford Univ. Press, London, 1939.

Department of Mathematics, University of Illinois, Urbana, Illinois 61801 